\title{
Status of JENDL
}

Osamu Iwamoto $^{1, *}$, Nobuyuki Iwamoto $^{1}$, Keiichi Shibata ${ }^{1}$, Akira Ichihara $^{1}$, Satoshi Kunieda ${ }^{1}$, Futoshi Minato ${ }^{1}$, and Shinsuke Nakayama ${ }^{1}$

${ }^{1}$ Nuclear Data Center, Japan Atomic Energy Agency, Tokai-mura, Ibaraki 319-1195, Japan

\begin{abstract}
Recent progress and future plan of the JENDL project are summarized. Two special purpose files were released recently. One is Photo-nuclear Data File 2016 (JENDL/PD-2016) which contains the data of photo-nuclear reaction cross sections covering a wide area of the nuclear chart. The other one is the JENDL Activation Cross Section File for Nuclear Decommissioning 2017 (JENDL/AD-2017) which provides production cross sections of radioactive nuclei by neutrons. A special purpose file JENDL/ImPACT-2018 dedicated to transmutation of long-lived fission products will be released in 2019. Regarding the general-purpose file, we are preparing to release the next version, which would be made available by 2022 as JENDL-5. Evaluation efforts on nuclides across wide range of nuclei form light to heavy have being made. The first test version JENDL-5 1 was created in 2018. The evaluated data will be updated on the basis of benchmark tests on reactor criticalities and neutron shieldings.
\end{abstract}

\section{Introduction}

More than 40 years have passed since the first version of Japanese Evaluated Nuclear Data Library (JENDL) was released in 1977. JENDL-1 was motivated to contribute to fast reactor development.[1] The latest version of the general purpose file JENDL-4.0 released in 2010 was developed to meet the needs of innovative reactors, use of high burn-up and MOX fuels, and so on.[2] JENDL-4.0 was focusing on improvement of data for fission products and minor actinides as well as enhancement of covariance data.

In addition to the general purpose file which targets reactor calculation, after the release of JENDL-2,[3] development of special purpose files was started to meet the needs from other than nuclear reactor analysis. As the first special purpose files, JENDL Dosimetry File 91[4] and JENDL Gas-Production Cross Section File 91[5] were released in July 1991. Other special purpose files were also actively developed and the 16 files were released by 2016 when the previous international nuclear data conference ND2016 was held.

After that, three special purpose files have been made regarding to photo-nuclear reaction, activation cross section and nuclear transmutation of radioactive wastes. The first one is Photonuclear Data File 2016 (JENDL/PD2016) and the second one is the JENDL Activation Cross Section File for Nuclear Decommissioning 2017 (JENDL/AD-2017). The last one is the LLFP Transmutation Cross Section File (JENDL/ImPACT-2018). Development of these files have been conducted in connection with the IAEA international collaboration on photonuclear data library, collaborative research on decom-

*e-mail: iwamoto.osamu@jaea.go.jp missioning with Japaneses atomic power company and the governmental funded project concerning transmutation of radioactive wastes, respectively.

Regarding the general purpose file, nuclear data evaluations are in progress towards the release of the next version JENDL-5 which is planned to be available by 2022 . In addition to meeting the needs from various field application, JENDL-5 is aiming to contribute to the existing important issues on nuclear energy in Japan such as treatment of nuclear waste and increase of nuclear safety. The evaluation works are in progress across wide range of nuclides from light to heavy ones based on currently available experimental data including new measurments by ANNRI at J-PARC especially for minor actinides. The number of nuclides will be increased by evaluating the data especially for stable nuclides which is lacked in JENDL-4.0. The first test version of the JENDL-5 called JENDL- $5 \alpha 1$ has been created by updating neutron reaction data for about 100 nuclides. Regarding thermal scattering law data for light water, a new Japanese evaluation is adopted. The benchmark tests for reactor criticality and neutron shielding are being performed to make feedback to the evaluations.

This article describes the status of JENDL project with respect to the development of the evaluated nuclear data libraries.[6]

\section{JENDL Special purpose file}

\subsection{JENDL/AD-2017}

JENDL Activation Cross Section File for Nuclear Decommissioning 2017 (JENDL/AD-2017) was released in March 2018. JENDL/AD-2017 was developed to meet the needs of radioactive inventory evaluation in decommissioning of light water reactors. The 211 nuclides regarded 
as important in inventory evaluation were selected. For the nuclides which possibly contribute to the production of concerned RIs, their cross sections were adopted form the existing libraries of JENDL-4.0 and JENDL/A-96 with or without modifications depending on their agreements with experimental data. In addition, new evaluations of the cross sections were also performed for the sake of both of the activation file and the general purpose file i.e. JENDL5. Isomer production cross sections, which is not usually included in the general purpose file, are important in the case of the activation file. Basically, they were consistently evaluated with the other cross sections by optimizing the model parameters so as to reproduce experimental data in high energy regions where the nuclear reaction model code was applied to the evaluations. In the resonance region, isomer production ratios were given referring to the experimental data at the thermal neutron energy if available.

JENDL/AD-2017 includes the cross sections of neutron reaction on 311 nuclides up to $20 \mathrm{MeV}$. Proton reaction data on ${ }^{56} \mathrm{Fe}$ are also contained. The pointwise data at two temperatures of $0 \mathrm{~K}$ and 293.6 are provided as JENDL/AD-2017.

Figure 1 shows cross sections of ${ }^{13} \mathrm{C}(\mathrm{n}, \gamma)$ reaction which is important for activation inventory evaluation because it produce long lived radio isotope ${ }^{14} \mathrm{C}$. Below $1 \mathrm{keV}$ of neutron incident energies, the cross section was evaluated based on Mughabghab's recommendation[7] and similar to other libraries, but evaluations deviate each other above it where Maximilian and spectrum averaged cross section measurements exist. The figure suggests that the present result, which includes direct neutron capture contribution, gives better consistency with the measurements in a range of 10 to $100 \mathrm{keV}$.

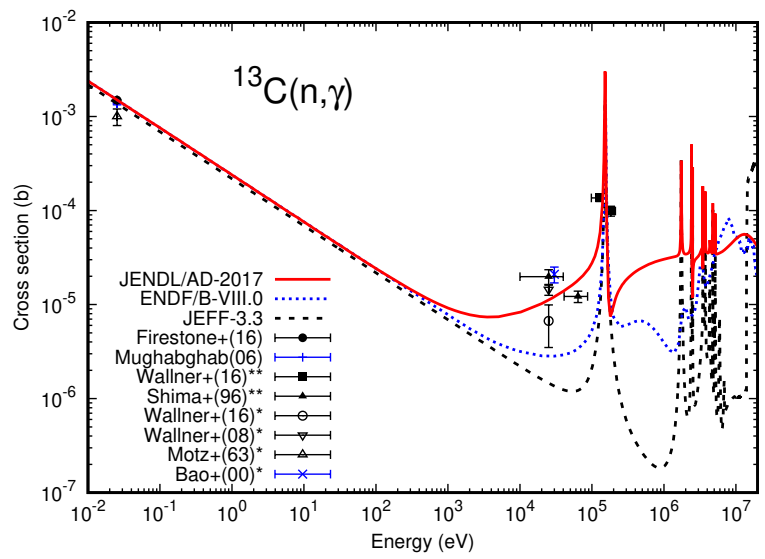

Figure 1. Neutron capture cross section of ${ }^{13} \mathrm{C}$. Evaluated cross sections are shown by lines. Experimental and recommended data are given by black and blue symbols, respectively. Authors with "**" and "**" indicate Maximilian and spectrum averaged cross sections, respectively.

\subsection{JENDL/PD-2016}

JENDL Photonuclear Data File 2016(JENDL/PD-2016) was released in December 2017. JENDL/PD-2016 con- tains data which are needed in $\gamma$-ray transportation simulations on accelerators, nuclear reactors, radiation, radiation exposure and so on. To cover wide areas of applications, the number of the included nuclei has been largely increased from the previous version JENDL/PD-2004.[8]

JENDL/PD-2016 includes photo-nuclear data for 2681 nuclides from $\mathrm{H}(\mathrm{Z}=1)$ to $\operatorname{Lr}(\mathrm{Z}=103)$. The full data of 2681 nuclides are stored in the expanded version. The standard version which consists of 181 nuclei along the beta-stability line was also prepared for convenience.

The file contains reaction cross sections: photoabsorption, photo-fission, particle and residual-nuclide production, and energy-angle distribution of light particle emission. The evaluation has been performed by using several nuclear reaction codes such as $\operatorname{CCONE}[9,10]$ and ALICE-F [11] at photon energies up to $140 \mathrm{MeV}$. The CCONE code was used mainly for the evaluation of nuclei along beta-stability lines and ALICE-F was applied to the evaluations of various nuclei including very unstable ones.

Figure 2 shows the cross section of one neutron emission from $\gamma$-ray induced reaction on ${ }^{100} \mathrm{Mo}$. The data of JENDL/PD-2016 was evaluated with the newest experimental data of Utsunomiya et al.[12] around the threshold energy of the reaction. As seen in the figure, the evaluated data of JENDL/PD-2016 shows good agreement with the experimental data.

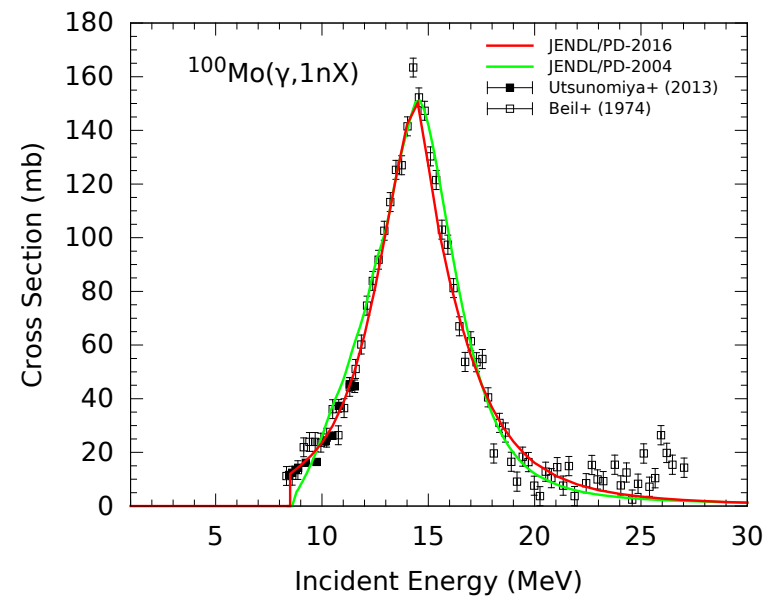

Figure 2. One neutron emission cross section of photon induced reaction on ${ }^{100} \mathrm{Mo}$. Red and green lines shows the evaluated data of JENDL/PD-2016 and JENDL/PD-2004, respectively. Experimental data are indicated by symbols.

In the collaboration with the IAEA photonuclear data activity, revisions of the data in JENDL/PD-2016 are in progress. They will be released as JENDL/PD-2016.1 around early 2020 .

\subsection{JENDL/ImPACT-2018}

A research program of "Reduction and Resource Recycling of High-level Radioactive Wastes through Nuclear Transmutation" was conducted to study a possible way to reduce long-lived fission products (LLFPs) under the ImPACT project in Japan. The program has a wide spec- 
trum on studies concerning from measurements of cross sections of nuclear reactions to concept design of transmutation system.

JENDL LLFP transmutation cross section File 2018 (JENDL/ImPACT-2018) has been developed in this program. As the program targeted effective use of accelerators for the nuclear transmutation, reliable data for LLFP and second products of transmutation were required across the wide energy range. JENDL/ImPACT-2018 intended to provide the reliable data of the proton and neutron induced reactions up to $200 \mathrm{MeV}$. To improve the accuracy of the data on LLFP whose experimental data are very limited, the results deduced from microscopic theories on nuclear structure have been widely used in evaluation with the reaction model code CCONE[9, 10] which has been applied in JENDL evaluation. In addition to use of $\gamma$-ray strength functions deduced from the modern nuclear theoretical calculations, a new level density formulation was developed and incorporated in CCONE. This new level density was developed by parameterizing in a hybrid way of spherical and deformed nuclei and their deformation properties were taken from microscopic theoretical calculations.[13] New experimental data of LLFPs obtained at the RIKEN RIBF facility using inverse kinematics were applied to the optimization of the parameter in preequilibrium exciton model. It gave significant improvement in the agreements with the experimental data of production cross sections over the wide range of produced isotopes.

JENDL/ImPACT-2018 provides the nuclear data regarding nuclides production and light particle emission for proton and neutron induced reactions on 163 nuclides which could be produced in transmutation of LLFPs of ${ }^{79} \mathrm{Se},{ }^{93} \mathrm{Zr},{ }^{107} \mathrm{Pd}$ and ${ }^{135} \mathrm{Cs}$. It will be released in 2019.

\section{JENDL general purpose file}

To contribute the acceleration of nuclear innovation on backend as well as various applications, development of the next version of general purpose file JENDL-5 is in progress. Aiming at covering the various needs in the wide range of applications, neutron reaction data for all stable isotopes are being planned to be included. To increase usability, it is intended to incorporate special purpose files into the general purpose file. In this direction, isomer production cross sections, which has been given in the activation cross section file, will be merged in JENDL-5. That would give the possibility for users to calculate neutron transportation and radioactive inventory evaluation with the single nuclear data library. The fission product yield and decay data also will be updated from the previous versions JENDL/FPY-2011 and JENDL/FPD-2011. The development of the decay data are in progress. The new decay data will include the decayed neutron spectra, which is not given in the JENDL/FPD-2011, incorporating with the theoretical calculations of microscopic nuclear structure theory for $\beta$-decay and statistical decay model for neutron emission.

We are planning to make revisions of data across a wide range of nuclides from light to heavy ones. For evaluation of the light nuclei resonances, a new code for R- matrix analysis has been developed. For minor actinides, cross section data in resonance region were intensively measured with ANNRI at J-PARC and they have been used to revise the resonance parameters. For major actinides, the fast neutron fission cross sections have being evaluated by the simultaneous evaluation method which was applied to previous JENDL evaluations.

The first test version JENDL-5 1 was created in 2018. JENDL- $5 \alpha 1$ contains updated and newly evaluated data for the isotopes of $\mathrm{Ga}, \mathrm{Zr}, \mathrm{Nb}, \mathrm{Tc}, \mathrm{Ru}, \mathrm{Sb}, \mathrm{Te}, \mathrm{I}, \mathrm{Pr}, \mathrm{Gd}$, Er, Ta, Re, Pt, Hg, Tl, U, Pu, and Am. For others, the data of JENDL-4.0 was adopted. A new thermal scattering law data for light water evaluated in Japan was tested with JENDL-5 $\alpha 1$.

Covariance data are planned to be enhanced in JENDL-5. Structure material and light nuclei are main targets of covariance evaluation in JENDL-5, in which the least-squares method would be applied based on the experimental data used in the evaluation. Charged particle induced reactions will be newly included in JENDL-5. JENDL-5 is planned to be released in FY2021.

\subsection{Light nucleus}

The reaction data on light nuclei are important for various applications such as nuclear reactors, medical use of radiation, astrophysics etc. However, most of data in JENDL4.0 were not updated when it was released. For the evaluation of resonance of light nuclei, a new R-matrix code has been developed[14] and has been applied to the evaluation of ${ }^{15} \mathrm{~N},{ }^{16} \mathrm{O}$ and ${ }^{19} \mathrm{~F}$.[15, 16] Preliminary evaluated data were obtained from the simultaneous analysis using cross sections of multiple reaction channels which lead to the same compound nucleus.

Figure 3 shows neutron total cross section of ${ }^{15} \mathrm{~N}$ which would be used as a fuel material of the accelerator driven system. The evaluated result is compared with the experimental data and JENDL-4.0. Agreement with experimental data is improved from JENDL-4.0 especially around $3 \mathrm{MeV}$ of neutron incident energy.

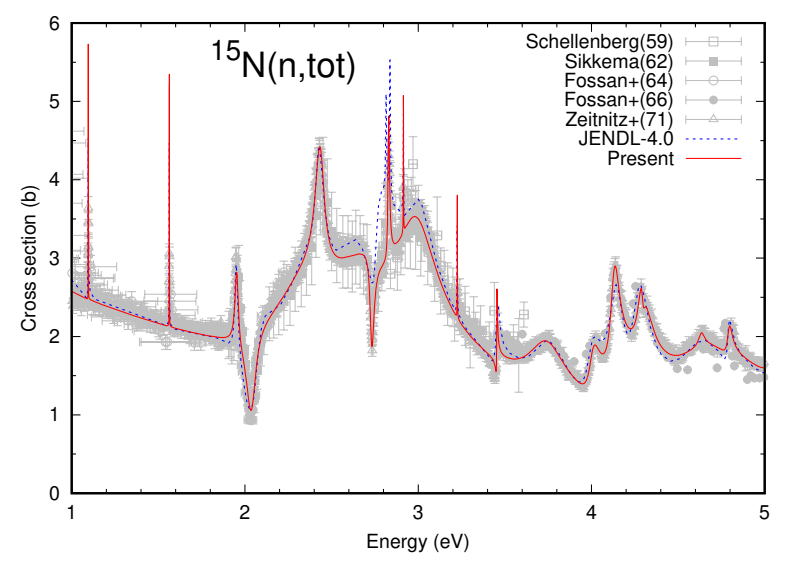

Figure 3. Neutron total cross section of ${ }^{15} \mathrm{~N}$. New evaluation and JENDL-4.0 were shown by red solid and blue dashed lines, respectively. 


\subsection{Structural material}

Revision of structure material data is one of main targets of JENDL-5, because their updates were limited when JENDL-4 was being developed in spite of their importance.

The ${ }^{93} \mathrm{Nb}$ isotope is used in high-melting point alloys and is a constituent of the superconductor in fusion reactors. Cross sections for ${ }^{93} \mathrm{Nb}$ isomer productions are important as a reactor dosimeter and a neutron flux monitor. The neutron reaction data on ${ }^{93} \mathrm{Nb}$ have been evaluated by $\mathrm{CCONE}[9,10]$ by optimizing the nuclear model parameters so as to reproduce the experimental cross sections for isomer production and total reaction simultaneously.[17] Figure 4 shows present results of ${ }^{93} \mathrm{Nb}(n, 2 n)$ cross section with other evaluations and experimental data. The present result agrees with the experimental data well for both of total reaction and isomer production.

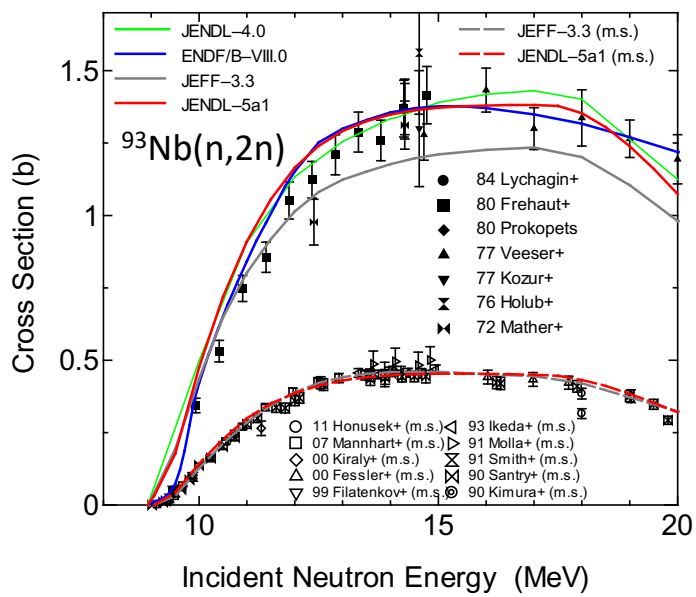

Figure 4. Cross section of ${ }^{93} \mathrm{Nb}(\mathrm{n}, 2 \mathrm{n})$. Solid and dashed lines mean total amount of $(n, 2 n)$ cross section and isomer production cross section in recent evaluated libraries, respectively. The present results (JENDL-5a1) is shown by red lines. Experimental data are shown by Various symbols.

The neutron reaction data on $\mathrm{Zr}$ and $\mathrm{Cu}$ have also been evaluated using CCONE taking account of available experimental data. Their results have already published.[18, 19]

\subsection{Fission products and medium heavy nuclides}

In the development of JENDL-4.0, most of FP resonance parameters were revised. However, among the $215 \mathrm{nu}-$ clides with $Z=30$ to 68 regarded as FPs in JENDL-4.0, the fast neutron cross sections for the 170 nuclides were still remained unchanged. Because in addition to the accumulation of new experimental data, the modern methods developed for the JENDL-4.0 evaluation would increase reliability on prediction of cross sections, it is desirable to update unrevised data using them. The nuclear data of FP isotopes of $\mathrm{Ga}, \mathrm{Zr}, \mathrm{Nb}, \mathrm{Tc}, \mathrm{Ru}, \mathrm{Sb}, \mathrm{Te}, \mathrm{I}, \mathrm{Pr}, \mathrm{Gd}$ and $\mathrm{Er}$ have been evaluated and their ENDF-6 formatted files are created.[17, 18, 20-27]
For the isotopes of $\mathrm{Ta}, \mathrm{Hg}, \mathrm{Tl}$ and $\mathrm{Bi}$, the cross sections have been revised, and the Pt isotopes data missed in JENDL-4.0 have been newly evaluated.[28-30] The data of ${ }^{209} \mathrm{Bi}$ which were partly revised for JENDL-4.0 have been fully re-evaluated because they are important for accelerator driven system for minor actinides transmutation. The evaluated cross section of ${ }^{209} \mathrm{Bi}(\mathrm{n}, 2 \mathrm{n})$ reaction is shown in Fig. 5. The present result agrees well with experimental data in the whole evaluated energy range.

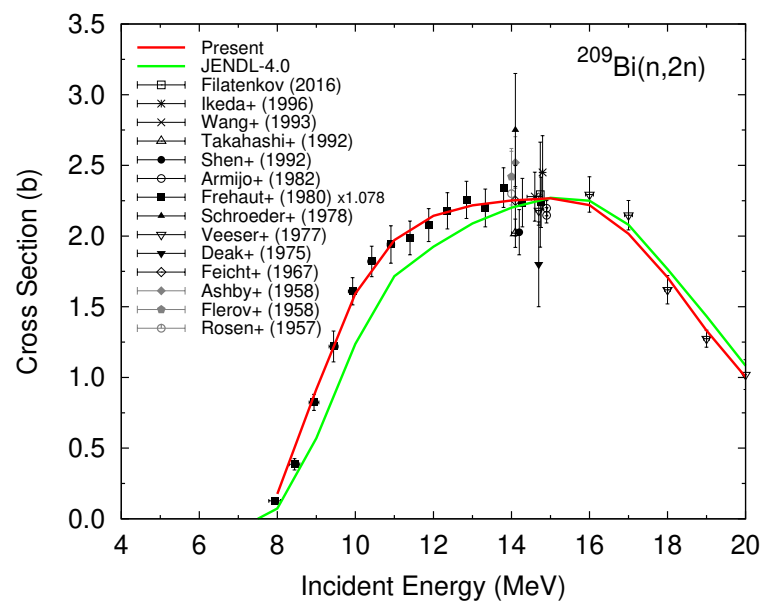

Figure 5. Cross section of ${ }^{209} \mathrm{Bi}(\mathrm{n}, 2 \mathrm{n})$. New evaluation and JENDL-4.0 are shown by solid red and dashed green lines, respectively.

\subsection{Actinide evaluation}

New data for minor actinides (MAs) were intensively measured under the AIMAC project which was intended to improve accuracy of MA nuclear data.[31] The time-of-flight data were obtained for neutron transmission and capture reaction of Am isotopes using ANNRI in J-PARC [32, 33]. From the analysis of those data, the preliminary results of resonance parameters of ${ }^{241,243} \mathrm{Am}$ were obtained with the REFIT code. Figure 6 shows the fitted results of ${ }^{243} \mathrm{Am}$. The three capture yield data with different amounts of samples and one transmission data were used for the analysis. The cross sections of JENDL-4.0 are also shown in the figure for comparison. While some deviations are seen for JENDL-4.0 evaluation, the present results show good consistency with all measured data.

For test library JENDL-5 $\alpha 1$, CIELO evaluations [34] of resolved resonance parameters of major actinides have been adopted. Simultaneous evaluation of the fission cross sections in fast neutrons energy region for 6 isotopes of $U$ and $\mathrm{Pu}$ are in progress. Their preliminary results are also included in JENDL- $5 \alpha 1$.

\subsection{Thermal scattering law data}

Thermal scattering law data of JENDL-4.0 were taken from ENDF-B/VII.0 or ENDF-B/VI.8. After the release of JENDL-4.0, Abe et al. published a new evaluation 


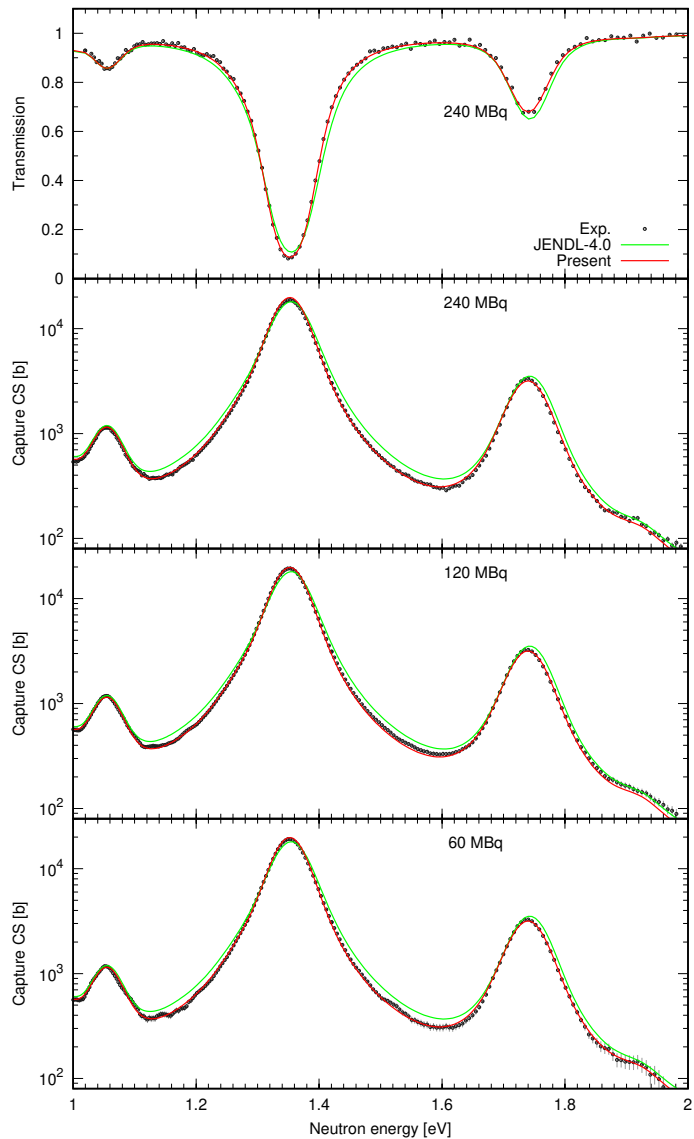

Figure 6. Cross section of ${ }^{243} \mathrm{Am}$. New evaluation and JENDL4.0 are shown in red and green lines, respectively. Experimental data are shown by dots. The activities in MBq indicate amounts of the samples used in the measurements.

for light water based on molecular dynamics.[35] It was adopted for JENDL- $5 \alpha 1$. Concerning the total cross section, overestimation seen in JENDL-4.0 below around 1 meV has been significantly improved. The data around the thermal neutron energy region were also revised. It would considerable impact on benchmark calculations on light water reactors.

\subsection{Deuteron data}

To extend the applicability of the general purpose file, JENDL-5 is planed to include not only the neutron induced reaction but also light charged particle reactions. Deuteron induced reaction is important as accelerator-based neutron source and their accurate reaction data are required for design of the system. The evaluation of the deuteron data is in progress.

The code system DEURACS has been developed for evaluation of deuteron induced reaction data.[36, 37] DEURACS takes account of the breakup processes of deuteron by the continuum-discretized coupled-channels theory and Glauber model for elastic and nonelastic breakup processes, respectively, and the preequilibrium and the compound processes are also considered in consistent manner with the breakup process by the CCONE code. DEURACS results show good agreement with the experimental data of neutron emission cross sections as well as residual nucleus productions. This consequence suggests that the proper treatment of the breakup process would be essential to describe deuteron induced reactions.

Figure 7 shows the emitted neutron energy spectrum at forward angles of 0 and 15 degrees from ${ }^{9} \mathrm{Be}(d, x n)$ reaction at the incident energy of $102 \mathrm{MeV}$. It is clearly seen the excellent agreement of the calculated results with the experimental data especially around the peaks at a half of the incident energy i.e. $\sim 50 \mathrm{MeV}$ which were predominantly created from the neutron by the deuteron breakup process. It should be noted that the calculations are not fitted to the experimental data.

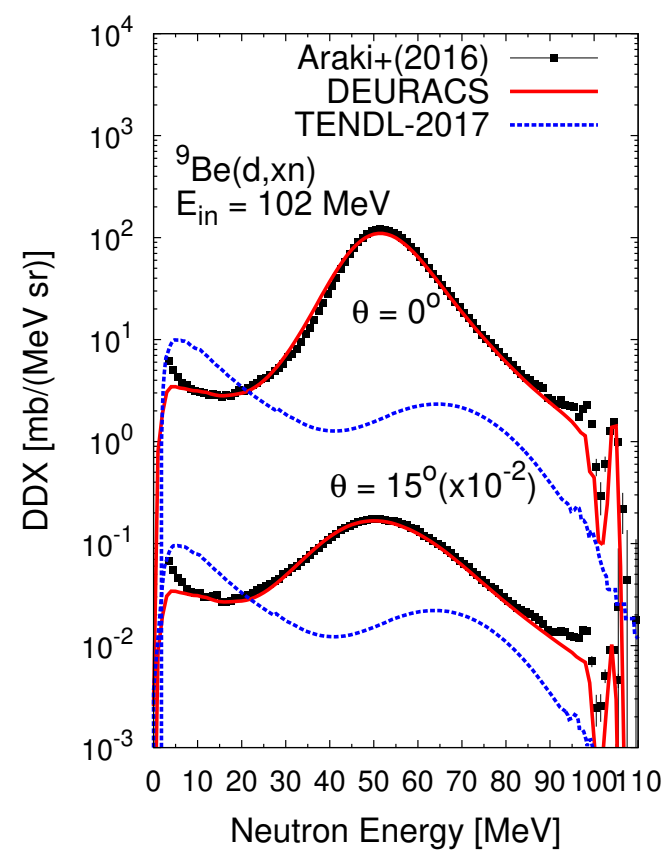

Figure 7. Double differential cross sections of ${ }^{9} \mathrm{Be}(d, x n)$ reaction. Results of DEURACS calculation (red line) are compared with evaluation of TENDL (dotted blue line) and experimental data (black dots).

\section{Conclusion}

Two special purpose files for activation cross section(JENDL-AD/2017) and photonuclear data file (JENDL/PD-2016) were released recently. A new special purpose file for LLFP transmutation (JENDL/ImPACT2017) will be released in 2019. Next version of general purpose file JENDL-5 is under development. The evaluation of cross section is in progress and the first test version JENDL- $5 \alpha 1$ has been created. Revision will be done on the basis of feedback from benchmark results of the JENDL- $5 \alpha 1$. For JENDL-5, evaluation of covariance data will be proceeded and fission product yields evaluated at Tokyo Tech will be adopted. In addition decay data will be updated for JENDL-5. JENDL-5 is planed to be released in FY 2021. 


\section{Acknowledgement}

This work was partly funded by the ImPACT Program of the Council for Science, Technology and Innovation (Cabinet Office, Government of Japan).

\section{References}

[1] S. Igarasi et al., JAERI 1261, Jpan Atomic Energy Research Institute (1979)

[2] K. Shibata et al., J. Nucl. Sci. Technol. 48, 1 (2011)

[3] S. Igarasi et al., J. At. Energy Soc. Jpn, 26, 191 (1984)

[4] M. Nakazawa et al., JAERI 1325, Jpan Atomic Energy Research Institute (1992)

[5] T. Nakagawa et al., JAERI-M 92-076, Jpan Atomic Energy Research Institute (1992)

[6] JENDL Japanese Evaluated Nuclear Data Library, https://wwwndc.jaea.go.jp/jendl/jendl.html

[7] S. F. Mughabghab, "Atlas of Neutron Resonances, Fifth Edition: Resonance Parameters and Thermal Cross Sections. Z=1-100”, Elsevier Science (2006).

[8] N. Kishida et al., Proc. of International Conference on Nuclear Data for Science and Technology, Santa Fe, New Mexico, USA, Sep. 26 - Oct. 1, 2004, 1, p.199 (2004)

[9] O. Iwamoto, J. Nucl. Sci. Technol. 44, 687 (2007)

[10] O. Iwamoto et al., Nucl. Data Sheets 131, 259 (2016)

[11] T. Fukahori, Proc. of the Specialists' Meeting on High Energy Nuclear Data, Tokai, Japan, 1991, JAERI-M 92-039, pp.114-122 (1992)
[12] H. Utsunomiya et al., Phys. Rev. C, 88, 015805 (2013)

[13] N. Furutachi, F. Minato, O. Iwamoto, J. Nucl. Sci. Technol. 56, 412 (2019)

[14] S. Kunieda, EPJ Web of Conf. 146, 12029 (2017)

[15] S. Kunieda, et al., Nucl. Data Sheets, 123, 159 (2015)

[16] S. Kunieda, et al., Nucl. Data Sheets, 118, 250 (2014)

[17] A. Ichihara, J. Nucl. Sci. Technol., 53, 2049 (2016)

[18] A. Ichihara, J. Nucl. Sci. Technol., 55, 1087 (2018)

[19] S. Nakayama, J. Nucl. Sci. Technol., 55, 614 (2018)

[20] K. Shibata, J. Nucl. Sci. Technol., 50, 277 (2013)

[21] N. Iwamoto, J. Nucl. Sci. Technol., 49, 244 (2012)

[22] K. Shibata, J. Nucl. Sci. Technol., 50, 1177 (2012)

[23] K. Shibata, J. Nucl. Sci. Technol., 51, 425 (2014)

[24] K. Shibata, J. Nucl. Sci. Technol., 52, 490 (2015)

[25] K. Shibata, J. Nucl. Sci. Technol., 52, 1174 (2015)

[26] F. Minato, J. Nucl. Sci. Technol., 50, 873 (2013)

[27] K. Shibata, J. Nucl. Sci. Technol., 49, 824 (2012)

[28] K. Shibata, J. Nucl. Sci. Technol., 53, 957 (2016)

[29] K. Shibata, J. Nucl. Sci. Technol., 53, 1595 (2016)

[30] K. Shibata, J. Nucl. Sci. Technol., 54, 147 (2017)

[31] H. Harada et al., EPJ Web of Conf. 93, 06001 (2015)

[32] K. Terada et al., J. Nucl. Sci. Technol. 55, 1198 (2018)

[33] A. Kimura et al., J. Nucl. Sci. Technol. 56, 479 (2019)

[34] M.B. Chadwick et al., Nucl. Data Sheets, 148, 189 (2018)

[35] Y. Abe, T. Tsuboi, S. Tasaki, Nucl. Instrum. Meth. A 735, 568 (2014)

[36] S. Nakayama et al., Phys. Rev. C, 98, 044606 (2018)

[37] S. Nakayama et al., Phys. Rev. C, 94, 014618 (2016) 